\title{
THE DYNAMO EFFECT IN MAGNETOHYDRODYNAMIC
}

\section{ACCRETION ONTO A ROTATING BLACK HOLE}

\author{
MASASHI EGI AND AKIRA TOMIMATSU \\ CG lab. Department of Physics, Nagoya University, Chikusa- \\ ku, Nagoya 464-01, Japan
}

AND

MASAAKI TAKAHASHI

Department of Physics and Astronomy, Aichi University of Education, Kariya, Aichi 448, Japan

\section{Intrduction}

A rotaing black hole has interesting features which can't be seen in nonrotating cases. The most characteristic one is to induce differential rotaions at angular velocity $\omega$ in inertial frames. Astrophysically it might have various impacts on its surrounding accretion plasmas. In a stationary and axisymmetric magnetohydrodynamic picture, it enables to directly extract the rotation energy of the black hole in the form of the outgoing Poynting flux. This process needs a condition $\Omega_{F}\left(\Omega_{H}-\Omega_{F}\right)>0$ on the horizon $r=r_{H}$, where $\left.\Omega_{H} \equiv \omega\right|_{r_{H}}$, and $\Omega_{F}$ is the angular velocity of magnetosphere. However, it is not so clear how the extracted energy activates the magnetosphere. Recently, Kahnna and Camenzind (KC 1994,1995) proposed a possibility of a self-excitation mechanism of the electromagnetic fields, supported by a coupling between $\omega$ and the angular velocity of plasma $\Omega$, through the magnetic diffusivity $\eta$ of accretion plasmas. They called it $\omega \Omega$ dynamo and tried to confirm this effect by numerical simulations. However no such growing cases were found in the initial conditions employed in the simulations (Brandenbrug 1996, KC 1996).

We analytically try to this problem in order to get some criterions for the growing cases, using a reductive perturbation method (Egi et al. 1997). The background space-time assumed to be a Kerr geometry. Our basic equations are Maxwell equations and a generalized Ohm's law for the elec- 
tromagnetic field tensor $F^{\mu \nu}$ giving a four velocity $u^{\mu}$ of a plasma fluid and $\eta$. Furthermore we assume axisymmetric magnetosphere and neglect any back reactions to $u^{\mu}$ for mathematical simplicity.

\section{Perturbation Schemes}

If the observed $\mathrm{X}$-ray variability is related to the magnetic diffusivity, then we suspect $\epsilon \equiv \eta / r_{H} \ll 1$, because its time-scale is much larger than the dynamical one. $\omega \Omega$ coupling must be strongest just on the horizon, so we focus our attention on a narrow boundary layer $x \equiv\left(r-r_{H}\right) / r_{H} \sim \mathcal{O}(\epsilon)$ and expand the field components with small parameter $\epsilon$.

According to the sprit of a reductive perturbation method, we can reduce this problem to the eigen value problem for eigen functions $\Psi(x, \theta)$ and eigen value $\lambda$. Here, $\Psi$ is a $\phi$ component of the vector potential, that is, $F_{\mu \phi}=\partial_{\mu} \Psi$. We can know some topological informations from $\Psi$. The growth of $\Psi$ is equivalent to the growth of electromagnetic fields, then $\lambda$ corresponds to the growing rate of $\Psi$ in a unit $\epsilon \times$ advanced time.

\section{Results}

We investigate the eigen value problem in two given velocity profiles of plasma fluids, which seem to be realistic. One is (i) $u^{t}=E, u^{\phi}=L \sin ^{2} \theta$ and the other is (ii) $u^{t}=E \sin ^{2} \theta, u^{\phi}=L \sin ^{2} \theta$, where $E$ and $L$ are constants. The results are;

1. The case (i) including the velocity profiles of previous simulations ( $\mathrm{KC}$ 1996, Brandenbrug 1996), which corresponds to $E<0$. However we find $E<0 \Rightarrow \lambda<0$ which means there is no growing mode. This agrees with the simulations.

2. In both cases (i) and (ii), we find $\lambda>0$ and $L>0 \Rightarrow 0<$ $\Omega_{H}<\Omega_{F}$, which means that the growing mode needs the condition $\Omega_{F}\left(\Omega_{H}-\Omega_{F}\right)>0$, because we naturally suppose $L>0 \Rightarrow \Omega_{F}>0$ at the horizon. This condition just equals to the one for the outgoing Poyinting flux to be positive.

We think that the extracted energy in the form of Poynting flux must be deposited in surrounding magnetosphere through the dynamo action, and conclude this paper with a conjecture that the deposited energy must be finally released in forming the jets.

\section{References}

Kahnna, R., Camenzind, M. Astophys. J., 435, L129 (1994)

Kahnna, R., Camenzind, M. Astron. Astrophys., 307, 665 (1996)

Brandenbrug, A. (1995) Astrophys. J., 465, L115 (1996)

Kahnna, R., Camenzind, M. Astrophys. J., 313, 1028 (1996)

Egi, M., Tomimatsu, A.,Takahashi, M., Plasma Phys. Control. Fusion, 39, A177 (1997) 